\title{
SURFACE EMG CHANNEL SELECTION FOR THUMB MOTION CLASSIFICATION
}

\author{
WAN-FEN XU ${ }^{1,2}$, YIN-FENG FANG ${ }^{5}$, GONG-YUE ZHANG ${ }^{5}$ ZHAO-JIE JU ${ }^{5}$, GONG-FA LI ${ }^{1,2,3^{*}}$,
}

\author{
HONG-HAI LIU
}

${ }^{1}$ Key Laboratory of Metallurgical Equipment and Control Technology of Ministry of Education, Wuhan University of Science and Technology, Wuhan 430081, China;

${ }^{2}$ Research Center for Biomimetic Robot and Intelligent Measurement and Control, Wuhan University of Science and Technology, Wuhan 430081, China;

${ }^{3}$ Institute of Precision Manufacturing, Wuhan University of Science and Technology, Wuhan 430081, China;

${ }^{4}$ Hubei Key Laboratory of Mechanical Transmission and Manufacturing Engineering, Wuhan University of

Science and Technology, Wuhan 430081, China;

${ }^{5}$ School of Computing, University of Portsmouth, Portsmouth PO1 3HE, UK

E-MAIL: one_fen@foxmail.com, yinfeng.fang@port.ac.uk, gongyue.zhang@port.ac.uk, zhaojie.ju@port.ac.uk, ligongfa@wust.edu.cn, honghai.liu@port.ac.uk

\begin{abstract}
:
Simplifying the interaction between humans and computers has become intensively important. Hand gesture contains large amount information that can facilitate the communication among humans, and it can also be utilized to interact with external devices. As a result, this study aims to decode the different hand gestures from sEMG signal. The thumb plays the most important role in hand-based object manipulation, such as touch screen control for smart phones, for which many thumb-based hand involved. Therefore, studying the relationship between EMG signals and the thumb movement has certain value for the future human-computer interaction. In this paper, we focus on the identification of electrode position. The signal from which is not so related to the thumb movement, and thus these sEMG channels can be reduced. In the experiment, a 16-channels sleeve is utilized and a variance-based method was proposed to identify the redundant channels. It is found that there exist three common redundant channels across nine subjects., and all located at the inside of the forearm.
\end{abstract}

Keywords:

EMG; Thumb; Gesture Recognition

\section{Introduction}

According to the clinical statistics, the thumb is responsible for at least half percent of all the hand function, especially for object grasping. It can be concluded that the thumb's movement affects the gesture so much, and further the pose of thumb affects the accuracy of the capture obviously. [1].

The muscles related to thumb have two groups which are intrinsic muscles and extrinsic muscles. Intrinsic muscles are located in the hand, and the extrinsic muscles are located in the forearm, as shown in Fig.1. The muscles of the forearm are more fixed than the muscles on hand, we only study the surface EMG in the forearm.

TABLE 1. THUMB-RELATED MUSCLES [3]

\begin{tabular}{|c|c|}
\hline Muscle Type & Muscle Name \\
\hline \multirow{3}{*}{$\begin{array}{c}\text { Intrinsic } \\
\text { muscle }\end{array}$} & Abductor Pollicis Transversus (APT) \\
\cline { 2 - 2 } & Flexor Pollicis Brevis (FPB) \\
\cline { 2 - 2 } & Abductor Pollicis Brevis (APB) \\
\cline { 2 - 2 } & First Dorsal Interossus (FDI) \\
\cline { 2 - 2 } Muscle & Opponent Pollicis (OP) \\
\cline { 2 - 2 } & Flexor Pollicis Longus (FPL) \\
\cline { 2 - 2 } & Abductor Pollicis Longus (APL) \\
\cline { 2 - 2 } & Extensor Pollicis Longus (EPL) \\
\cline { 2 - 2 } & Extensor Pollicis Brevis (EPB) \\
\hline
\end{tabular}




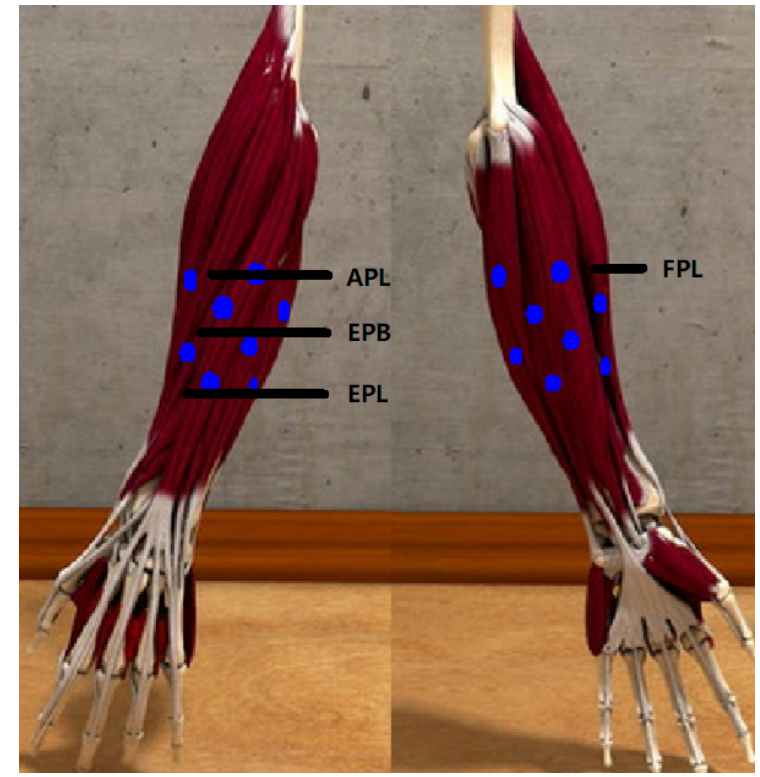

Fig.1 Extrinsic Muscle [2]

During the last decade, a large amount of researchers studied EMG signal based hand motion recognition, but most of them focused on the whole hand and few of them focused on the fingers. Xun Chen, and Z. Jane Wang have investigated the recognition accuracy affected by the features and the classification algorithms. The experiment used four channels to collect forearm sEMG signals from ten Chinese hand gestures. By the combination of traditional feature and the proposed classification algorithms, the hand movement recognition accuracy improved to more than 95\% [4]. Jongin Kim, Dongrae Cho, Kwang Jin Lee and Boreom Lee used sEMG signals to recognize the pinch-to-zoom gesture by distance [5]. Nor Anija Jalaludin, Abu Ubaidah Shamsudin, Shahrul Na' im Sidek et al designed a device to detect the sEMG and the force of the thumb to establish a sEMG model on the relation between thumb angle and force [6].

The current research focused on the study of sEMG channel selection from 16 sEMG channels, and it aimed to find some common channels that are redundant for thumb motion classification across different subjects. The identification of redundant electrode on a certain area of the forearm could provide a valuable reference for other related studies, and also can reduce the cost of sEMG signal acquisition device for external device interaction.

\section{Experiment}

\subsection{Experiment Design}

Two females and seven males volunteered in the experiment. All subjects are healthy and around 25 years old. The experiment refers to the measurement of five dynamic actions of the left hand's thumb which are up, down, left, right, and press (Fig. 2). For each subject, there was divided into five sessions. Every session is divided into five gestures, and each gesture repeated ten times, total 2250 time of gestures in the database. Every time of gesture lasted for 10 seconds which contained two periods. One is movement, and another is relaxation [7].

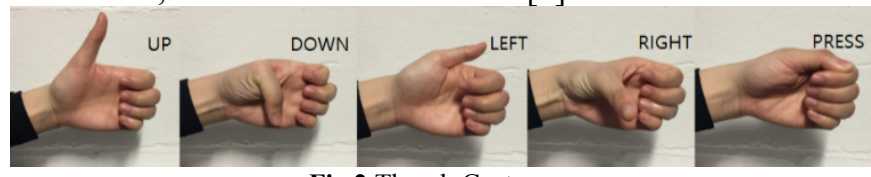

Fig.2 Thumb Gesture

\subsection{Hardware selection}

The EMG acquisition hardware is a 16-channel high temporal and spatial resolution sEMG collector with Bluetooth wireless communication and a sEMG sleeve with 16 dry electrodes [8]. The sEMG collector comes with a $50 \mathrm{HZ}$ comb filter and a $20 \mathrm{HZ}$ high-pass butter worth filter that could filter out most of the common noise and the dry electrode on the sleeve is uniformly distributed on the forearm.

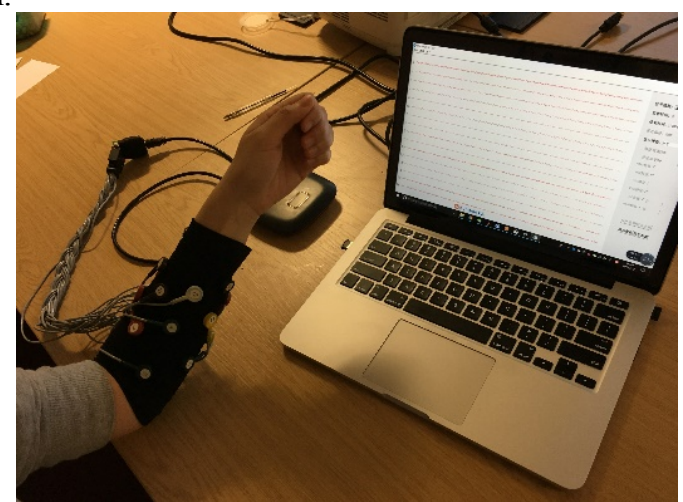

Fig.3 Hardware in Experiment

\subsection{Signal Processing}

Since we need to segment a whole dynamic gesture that the subject would inevitably make the signals inconsistencies, the raw signal needs to do some processing. 
a) Active Segment.

Each gesture was segmented into ten pieces, and each piece has ten seconds, forming ten cycles. Actions were started from the 5th second of each cycle and completed before the 10th second. The total process continued 100 seconds.

One can find that the inconsistency of the speed of each person's movement, for ensuring the integrity of the action, the starting point of the division is set to 4.8 seconds per cycle, and the end point of the division is the end point of the cycle [9].

b) Feature Extraction

The MAV feature reflects the mean value of the signal in a channel at intervals of several seconds. It reduced the dimension of the raw matrix and facilitates to obtain the variance in the later stage. In addition, three other time domain features are also extracted, including the number of slope sign changes feature (SSC), Window Length feature (WL), The number of zero crossings feature (ZC).

c) Calculate the Variance of Each Channel for Each action

Variance reflects the degree of deviation between the random variable and the expectation, and it reflects the activity of the random variable. The electrode which is more active means the more information in it. Thereby the variance indicates the usefulness of the electrode. In the forearm, there are only 4 muscles associated with the thumb, it reveals that not all external surfaces of the forearm will generate sEMG signal [10]. We suspect that the electrode which the variance is large indicates it is more active and more useful, whereas the electrode which the variance is small indicates it is more steady and more redundant.

$$
V A R=\frac{1}{N-1} \sum_{i=1}^{N} x_{i}^{2}
$$

For each piece of gesture, we calculate the variance for each channel after obtained the MAV feature and sorted by the value of the variance from large into small [11]. The higher the ranking is, the higher the weight assigned to the channel is.

Finally, we removed one subject respectively, and accumulated the remaining eight subjects' coefficients by channel. As showed in Fig.4, every channel has their own corresponding coefficient value. It can be assumed that the channel with the larger value is the more redundant, whereas the channel with the smaller value is the more useful.

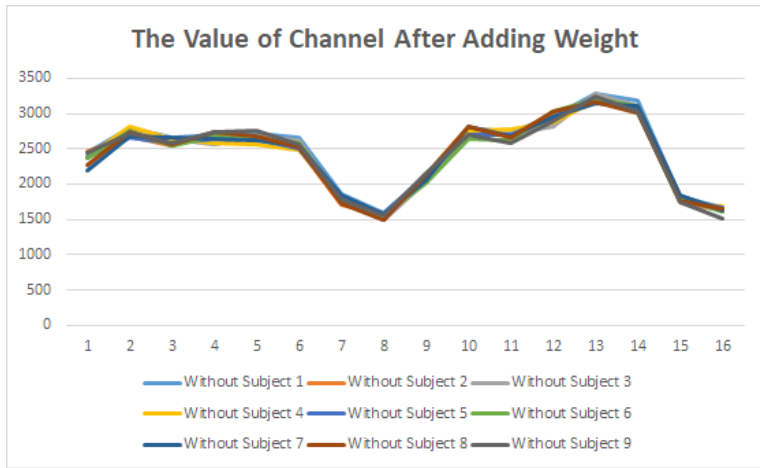

Fig.4 The Value of Channel After Adding Weight

\section{d) Finding Redundant Channels}

Arrange the value into 16 levels in order. Separate evaluating the channel values for each subject set and assign the level label. The label in this experiment is 16 different colors. Then choose their common redundant channel as the redundant channel [12]. Depending on Figure 5, the most redundant channel is number 13.

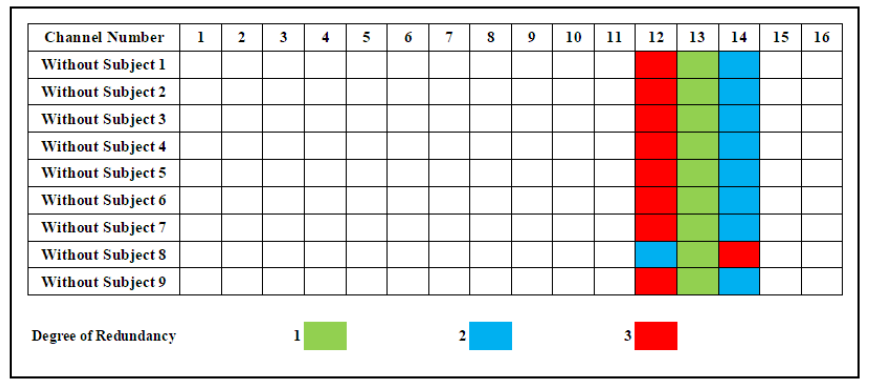

Fig.5 Most Redundant 3 Channels of Each Subject

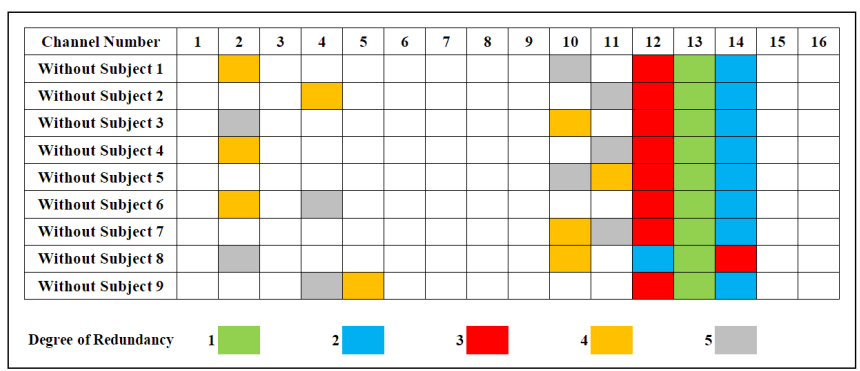

Fig.6 Most Redundant 5 Channels of Each Subject

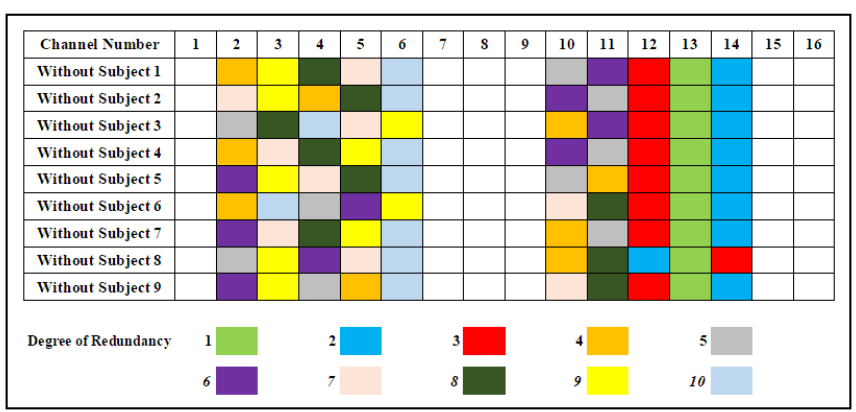


Fig.7 Most Redundant 10 Channels of Each Subject

Figure 6 shows that for the different subject, there are three same redundancy channels which are No. 13, No. 14 and No. 12, respectively, In the same reasoning, according to Figure 7, the most ten redundant channels are number 2 to number 6 and number 10 to number 14 .

e) Gesture recognition

By the algorithm of SVM to compare the data which after removing redundant channels and the data which have 16 channels, and calculating the recognition accuracy respectively [13].

According to the above three figures, there are three types of common redundant channels which are removing one channel, removing three channels, and removing ten channels.

The Accuracy Rate of SVM Classification Results

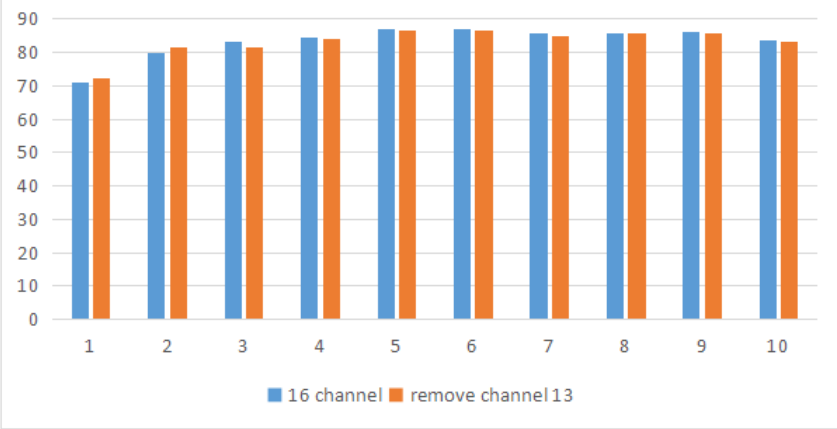

Fig.8 The Accuracy Rate of SVM Classification Result Without One Redundant Channel (\%)

The Accuracy Rate of SVM Classification Results

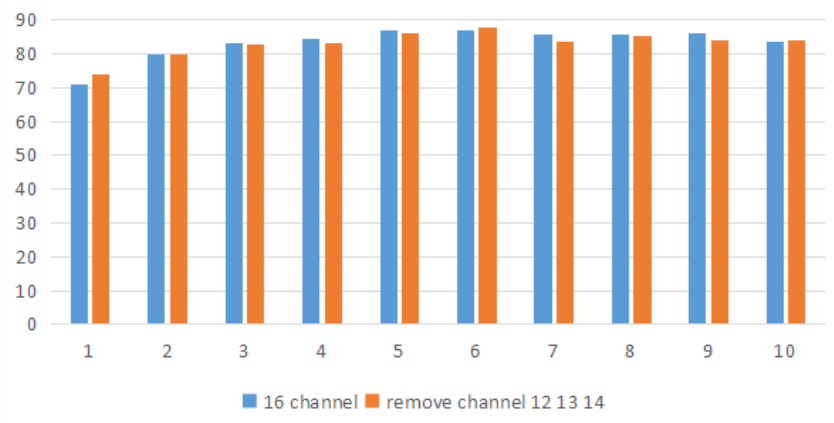

Fig.9 The Accuracy Rate of SVM Classification Result Without Three Redundant Channel (\%)

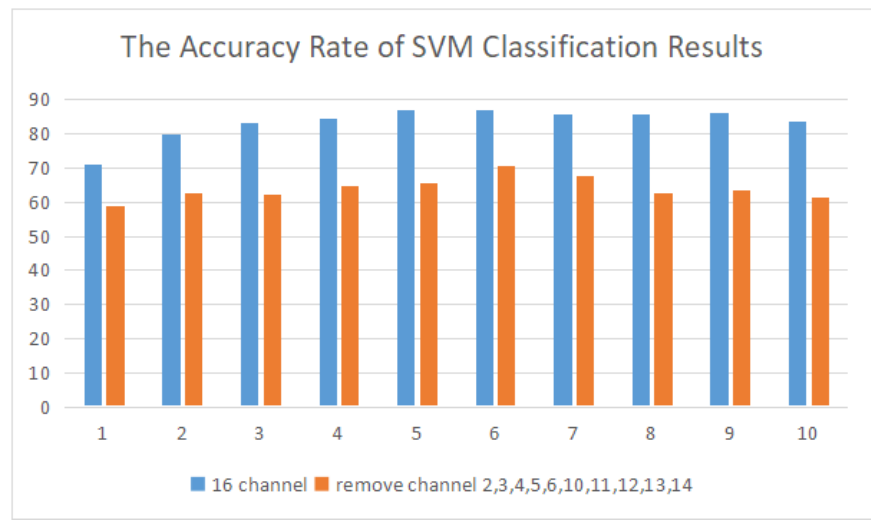

Fig.10 The Accuracy Rate of SVM Classification Result Without Ten Redundant Channel (\%)

The result shows that the recognition rate of 16 channels is $83.422 \%$, the recognition rate of removing one common redundant channel is $83.222 \%$, the recognition rate of removing three common redundant channels is $83.067 \%$, and the recognition rate of removing ten common redundant channels is $64 \%$.

\section{Conclude}

Since the redundant channel is based on the different subject set, we choose every eight channels to infer the unknown subject, and the conclusion is same. Therefore we can conclude that the redundant channel has a certain robustness [14]. There are only three situations to obtain for the redundant channels from this experiment, which are one channel, three channels, and ten channels respectively. Depending on Fig.8 and Fig.9, when removing one electrode or removing three electrodes, the recognition rate just changed little. When removing ten electrodes, the recognition rate drops rapidly. Even in some groups, removing the redundancy electrode could increase the recognition rate.

Compared with the anatomical map, the location of Extrinsic Muscle is not consistent with the location of electrodes which are number 12, number 13, and number 14 . Therefore, it can be concluded that the electrodes of number 12, number 13, and number 14 are redundant electrodes about the thumb, and it can be further explained that the area does not generate sEMG signal about the thumb.

In this experiment, we had confirmed the position where the forearm did not produce the electrical signal of the thumb by recognition of the movement of the thumb. But the sleeve used in this experiment had only 16 channels, the limitation of the electrodes resulted in redundant locations that were not specific enough [15]. It is obvious that further research will focus on high-density electrode 
sleeve to find more redundant areas locations in the forearm, further to invent the high-precision and low-cost sEMG device.

\section{Acknowledgements}

The authors would like to acknowledge the support from the Natural Science Foundation of China under Grant No. 51575412, 51575338, 51575407 and 61733011, the Grants of National Defense Pre-Research Foundation of Wuhan University of Science and Technology under Grant No. GF201705, the EU Seventh Framework Programme (FP7)-ICT under Grant No. 611391, and the Research Project of State Key Lab of Digital Manufacturing Equipment \& Technology of China under Grant No. DMETKF2017003.

\section{References}

[1] Abdul Rahman Siddiqi, Shahrul Naim Sidek, Aida Khorshidtalab. Signal Processing of EMG Signal for Continuous Thumb-Angle Estimation[J]. IECON,2015, pp. 374-379.

[2] Yinfeng Fang, Zhaojie Ju, Xiangyang Zhu et al. Finger Pinch Force Estimation Through Muscle Activations Using A Surface EMG Sleeve On the Forearm[C]. IEEE International Conference on Fuzzy Systems, International Conference on Fuzzy System, 2014

[3] Muhammad Rozaidi Roslan, Shahrul Naim Sidek, Siti Fauziah Toha et al. Portable Thumb Training System for EMG Signal Measurement and Analysis[C]. International Conference on Computer \& Communication 978-1-5090-2427-8/16,2016

[4] Xun Chen, Z. Jane Wang. Pattern recognition of number gestures based on a wireless surface EMG system[J]. Biomedical Signal Processing and Control, 2013, pp. 184-192

[5] Jongin Kim 1, Dongrae Cho 2, Kwang Jin Lee et al. A Real-Time Pinch-to-Zoom Motion Detection by Means of a Surface EMG-Based Human-Computer Interface[J]. Sensors, 2015, 15, pp.394-407

[6] Nor Anija Jalaludin, Abu Ubaidah Shamsudin, Shahrul Na'im Sidek et al. Electromyography (EMG)-based Thump-tip Force Estimation for Prosthetic Thumb[C]. International Conference on Computer and Communication Engineering, 2012

[7] Abdul Rahman Siddiqi, Shahrul Nairn Sidek, Muhammad Rozaidi Roslan. EMG based Classification for Continuous Thumb Angle and Force Prediction[J]. International Symposium on Robotics and Intelligent Sensors, 2015, pp. 43-47

[8] A. Aranceta-Garza, H. Lakany, B. A. Conway. An investigation into thumb rotation using high density surface electromyography of extrinsic hand muscles[C]. International Conference on Systems, International Conference on Systems, 2013

[9] Donny Huang, Xiaoyi Zhang, T. Scott Saponas. Leveraging Dual Observable Input for Fine Grained Thumb Interaction Using Forearm EMG[J] pp. 523-528

[10] Jongin Kim, Dongrae Cho, Kwang Jin Lee et al. A Real-Time Pinch-to-Zoom Motion Detection by Means of a Surface EMG-Based Human-Computer Interface[J]. Sensors, 2015, vol. 15, pp. 394-407

[11] L R Quitadamo, F Cavrini, L Sbernini et al. Support vector machines to detect physiological patterns for EEG and EMG-based human-computer interaction: a review[J]. Journal of Neural Engineering,2017, pp. $14: 1-27$

[12] Abdul Rahman Siddiqi, Shahrul Naim Sidek. Estimation of continuous thumb angle and force using electromyogram classification[J]. International Journal of Advanced Robotic Systems, 2016, pp. 1-12

[13] Visakha K. Nanayakkara, Giuseppe Cotugno, Nikolaos Vitzilaios et al. The Role of Morphology of the Thumb in Anthropomorphic Grasping: A Review[J]. Frontiers in Mechanical Engineering, 2017, vol. 3, no.5, pp. 1-21

[14] Nor Anija Jalaludin, Abu Ubaidah Shamsudin, Shahrul Na'im Sidek et al. Electromyography (EMG)-based Thump-tip Force Estimation for Prosthetic Thumb $[\mathrm{J}]$. International Conference on Computer and Communication Engineering, 2012, pp. 3-5

[15] Yinfeng. Fang, Honghai. Liu, Gongfa. Li et al. A Multichannel Surface EMG System for Hand Motion Recognition[J]. International Journal of Humanoid Robotics, 2015,12 